medgen 2015 • 27:197-198

DOI 10.1007/s11825-015-0056-0

Online publiziert: 11. August 2015

(c) Springer-Verlag Berlin Heidelberg 2015

\title{
Editorial
}

gung, dass nur geschätzte $5 \%$ aller sog. „familiären Krebserkrankungen“ auf einer monogen vererbten Disposition beruhen, heißt dies, dass es sich theoretisch um etwa jede 60. Familie handelt. Auch wenn diese Zahlen nur sehr grobe Annahmen sind, zeigt dies die versorgungsmedizinische und damit verbunden gesundheitsökonomische Dimension der Thematik. Und genau hier liegt eines der Kernprobleme im Umgang mit den familiären Tumordispositionssyndromen: Es handelt sich um eines der größten Segmente im humangenetischen „Markt“. Dabei reicht dieser „Markt“ weit über die genetische Beratung und Diagnostik hinaus: Durch die prädiktive Testung werden langfristig gesunde und häufig noch junge Personen, die ein erhöhtes Tumorrisiko tragen, zur präventivmedizinischen Versorgung an klinische Einrichtungen gebunden, in denen von Gynäkologen, Internisten, Radiologen und anderen Disziplinen ein engmaschiges Vorsorgeprogramm durchgeführt wird. Zusätzlich führt die Einführung „individualisierter“ Therapien, seien es risikoadaptierte Regime oder echte „targeted therapies“, zunehmend zu einer prätherapeutischen genetischen Testung des Tumorgewebes (auch) auf solche Veränderungen, die mit einer hereditären Disposition für Tumorerkrankungen einhergehen.

In diesem nur grob skizzierten und sich rasant entwickelnden Spannungsfeld ist das vorliegende Themenheft zum familiären Brust- und Eierstockkrebs entstanden. Es wurde federführend gestaltet von Mitgliedern des „Deutschen Konsortiums für Familiären Brust- und Eierstockkrebs“. Dieses Konsortium wurde kurz nach der Entdeckung der beiden mit der monogenen Disposition für Brust- und Eierstockkrebs assoziierten Gene BRCA1 und BRCA2 im Jahre 1996 gegründet und wird seitdem kontinuierlich finanziell von der Deutschen Krebshilfe gefördert. Dabei war das überge- ordnete Ziel, bundesweit eine flächendeckende und qualitätsgesicherte Versorgung für Personen mit Verdacht auf eine erbliche Disposition für Brust- und Eierstockkrebs zu etablieren. Hinzu kommt ein breites Spektrum von erfolgreich bearbeiteten Forschungsprojekten des Konsortiums, das von der Identifizierung neuer, für Brust-/Eierstockkrebs disponierender Gene bzw. Genvarianten über psychoonkologische und Lebensstilfragestellungen bis hin zu Konzepten der Prävention und Vorsorge reicht. Für die humangenetische Beratung besonders wichtig ist die systematische Erhebung und Zusammenführung von Familien- und Mutationsdaten, welche wesentliche Informationen zur Interpretation von Sequenzvarianten und Berechnung von Risikoziffern bereitstellen. Den Erfolg des Konsortiums kann man daran ermessen, dass die erarbeiteten Konzepte von vielen Krankenkassen im Rahmen von Kooperationsverträgen mit den Zentren des Konsortiums umgesetzt und finanziert werden und dass die BRCA1- und BRCA2-Testung in den EBM und damit in die Regelversorgung übernommen wurde.

Doch genau dieser Erfolg, d. h. die erfolgreiche Überführung eines Forschungskonzepts in die Regelversorgung, ist zumindest für das Konsortium auch ein „Fluch“: Zahlreiche zusätzliche Anbieter der BRCA-Diagnostik verhindern nunmehr, dass das Konsortium eine vollständige populationsweite Erhebung zur Thematik weiterführen kann. Dies limitiert zukünftige wissenschaftliche Projekte und die Begleitforschung. Wichtiger aber ist wohl die Frage, ob die flächendeckende Zugänglichkeit der BRCA-Keimbahndiagnostik auch jenseits der Zentren des Konsortiums zu einem Verlust der Versorgungsqualität der Patienten und Ratsuchenden führt. Angesichts der umfassenden Akkreditierung humangenetischer Labore ist das in Bezug auf die eigentlichen Laborprozesse für die Keim-
Krebs auftitt. Selbst un "familiar 
bahnanalytik kaum zu erwarten. Sehr wohl müssen aber Bedenken hinsichtlich der Interpretation der Befunde und der diesbezüglichen Beratung bestehen, da die in den letzten 10 Jahren dank umfangreicher Forschungsförderung gesammelten Erfahrungen, Daten und Befunde momentan ausschließlich Mitgliedern des Konsortiums zur Verfügung stehen.

Aus dem oben Dargestellten wird ersichtlich, dass die in diesem Heft adressierte Thematik mit einer Reihe von sich z. T. komplementär gegenüberstehenden Interessen behaftet ist und behaftet sein muss. Dies betrifft sowohl wissenschaftliche als auch ökonomische und berufspolitische Aspekte. Die Sichtweisen z. B. bezüglich der Verfügbarkeit von Daten oder des Zugangs zu gewissen Versorgungsstrukturen können sich deutlich unterscheiden, z. B. entsprechend der Mitgliedschaft im Konsortium oder der Zugehörigkeit zu unterschiedlichen Fächern der Medizin. Dies hat zur Folge, dass für Koordinatoren des Themenbandes, Autoren der einzelnen Artikel und die involvierten Gutachter ein immanenter und wahrscheinlich nicht $\mathrm{zu}$ vermeidender Interessenkonflikt gegeben ist, der in einigen Aussagen dieses Heftes auch gut nachvollziehbar ist. Auch die Mitglieder der aktuellen und zukünftigen Schriftleitung, die ebenfalls zum Teil den Zentren des Konsortiums angehören (oder auch nicht), sind von diesem Interessenkonflikt nicht ausgenommen und möchten mit diesem Editorial explizit hierauf hinweisen. Auch wenn wir uns bemüht haben, auf eine objektive und informative Darstellung der Themen hinzuwirken, bleiben die in den Artikeln dargestellten Inhalte letztlich in der Verantwortung der Verfasser.

Und was ist nun das Fazit? Die Beratung, Diagnostik und Betreuung von Patienten mit erblichen Krebserkrankungen und deren Angehörigen ist eine interdisziplinäre Herausforderung, der sich die beteiligten Fachdisziplinen gemeinsam widmen müssen. Oberstes Gebot ist die Sicherstellung der qualitativ bestmöglichen und gut zugänglichen Versorgung der Patienten inkl. der Sicherstellung ihrer informationellen Selbstbestimmung. Dies kann weder durch ein Monopol auf Versorgung noch durch eines auf Daten erreicht werden. Stattdessen wird eine Kul- tur des Miteinanders der verschiedenen Fachdisziplinen und Beteiligten benötigt, wie es die ursprüngliche Idee des Konsortiums bei dessen Gründung war. Zugriff auf für den Patienten wesentliche Daten, zumal wenn sie in durch gezielte Förderung erst möglichen Forschungsprojekten generiert werden, sollte genauso selbstverständlich sein, wie umgekehrt Bereitstellung neu erhobener Daten - soweit es Datenschutz und Patienteneinwilligung erlauben. Miteinander muss aber insbesondere auch eine faire Finanzierung durch die Kostenträger eingefordert werden, welche sowohl Qualität als auch Weiterentwicklung sicherstellen, zum Wohle des Patienten. Vielleicht kann das vorliegende Heft dazu einige Anregungen geben.

\section{Die Schriftleitung}

Prof. Dr. med. Christian Kubisch, Hamburg (verantwortl.)

Prof. Dr. med. Reiner Siebert, Kiel

Prof. Dr. med. Ute Felbor, Greifswald

Prof. Dr. med. Michael Speicher, Graz

Prof. Dr. med. Dagmar Wieczorek, Essen

(CME-Schriftleiterin)

Prof. Dr. med. Tiemo Grimm

Prof. Dr. med. Klaus Zerres 